Estimation of Weibull Parameters $C_{0} N^{2}-680807--1$ from Failure Data

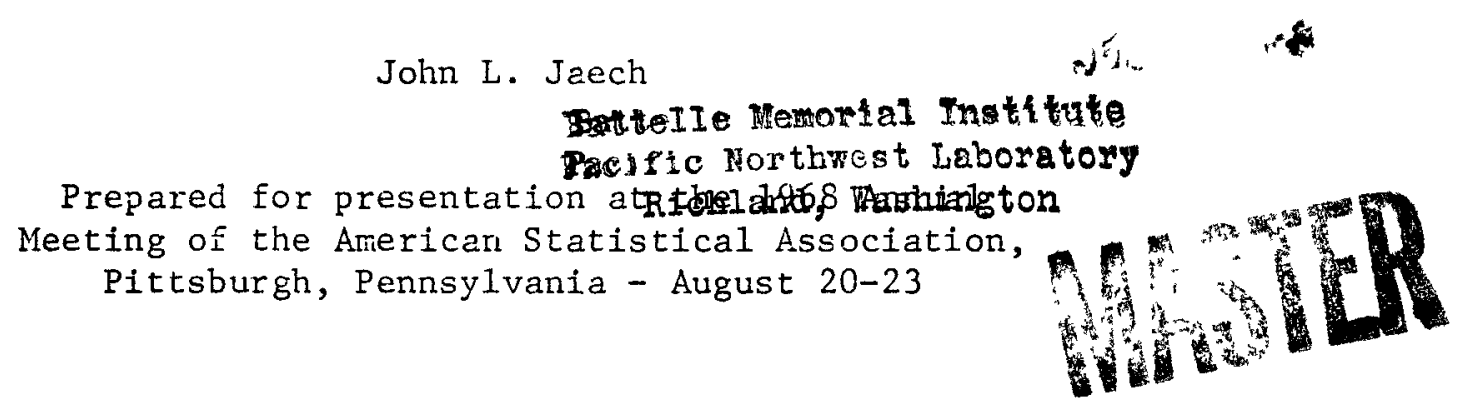

(1) Introduction

In a 1951 paper, Weibull [1] popularized a density function now bearing his name. He suggested a wide variety of situations in which this particular function provided a good description of the data. His examples included such random variables as the yield strength of a Eofors steel, the size distribution of fly ash, and the fiber strength of Indian cottun.

In recent years, the Weibull function has indeed been applied to a variety of problems. This is especially true in the field of life testing. Although much of the early work in life-testing was based on the exponentia: mode1, more recent attention has been directed toward using the more general Weibull model to describe failure data, since the exponential model has proven to be unrealistic in a number of situations.

This emphasis on the Weibull model has not extended over very many years. A bibliography compiled by Splitstone [2] in his 1967 Iowa State University Master's thesis on estimation of the Weibull parameters demonstrates that the emphasis is recent. Of the 25 references he 1 ists, only two were dated before 1959 and only 7.before 1963. Fourteen were dated 1965 or 1ater.

Judging by the dates of these publications, application of the Weibull model to reactor fuel element failures at Hanford represents somewhat of a pioneering effort. This model was first applied to such failure data in 1954, and has been used with success ever since. In addition to this application to fuel elements from plutonium production reactors, similar surcess has bəen.

This work was performed under contract number AT (45-1)]830 for the U. S. Atomic Energy Commission. 
achieved when describing fuel failure data from commercial power reactors. Methods of estimating the parameters of this distribution have been developed over the 15 years since the model was first applied at Hanford. These improvements are largely a result of increased computer capabilities which permits the use of estimation methods that were literally impossible to apply without such computational assistance. No doubt, increased computer capabilities have also contributed to other recent interest in the Weibull function as mentioned earlier, since many of the estimation procedures suggested by the various authors require large-scale computers.

In this paper, a method used to estimate the shape and scale parameters of the Weibull distribution as applied to reactor fuel element failures is discussed. The location parameter is assumed to be zero. The method of estimation may, of course, be used on other kinds of failure data as well.

\section{(2) The Data}

The data for which the estimation method was developed are grouped data. These are normally the way in which reactor fuel element failure data are made available. The data are characterized by large numbers of elements, and small numbers of failures.

The data appear as in Tabie (2.1)

Table (2.1)

\section{Failure Data}

$\begin{array}{cccc}\frac{\text { Time }}{0-\mathrm{T}_{1}} & \text { Number of elements } & \text { Number of losses } & \text { Number of failures } \\ \mathrm{T}_{1}-\mathrm{T}_{2} & \mathrm{~N}_{1} & \mathrm{~L}_{1} & \mathrm{r}_{1} \\ \mathrm{~T}_{2}-\mathrm{T}_{3} & \mathrm{~N}_{3} & \mathrm{~L}_{2} & \mathrm{r}_{2} \\ \vdots & \vdots & \mathrm{L}_{3} & \mathrm{r}_{3} \\ \mathrm{~T}_{\mathrm{k}-1}-\mathrm{T}_{\mathrm{k}} & \mathrm{N}_{\mathrm{k}} & \vdots & \vdots \\ & \mathrm{N}_{\mathrm{n}} & \mathrm{L}_{\mathrm{k}} & \mathrm{r}_{\mathrm{k}}\end{array}$


In this table $\mathrm{N}_{1}$ represents the total number of elements under study. In the $i^{\text {th }}$ time interval, $L_{i}$ elements are "lost", while $r_{i}$ failures are observed. A "lost" element, in the context of this example, is one which has either been discharged from the reactor at some time between $\mathrm{T}_{1-1}$ and $T_{1}$, or else is still in the reactor with an accumulated life which lies in this $i^{\text {th }}$ interval. $\mathrm{N}_{2}$, the number of elements present at the beginning of the second time interval, is clearly $\left(\mathrm{N}_{1}-\mathrm{L}_{1}\right)$, and, in general, $\mathrm{N}_{1}=$ $\left(N_{i-1}-L_{i-1}\right)$. (The number of losses, $L_{i}$, is defined to include the number of failures, $\left.r_{i}\right)$.

(3) Non-Parametric Estimation of the Failure Rate

For grouped failure data such as these, Kaplan and Meier [3] gave nonparametric procedures for estimating the failure rate. A simple procedure, called the actuarial method, consists of calculating the faiiure rate in each interval as

$$
\begin{aligned}
p_{i} & =r_{i} /\left(N_{i}-0.5 L_{i}\right) \\
& =r_{i} / m_{i}
\end{aligned}
$$

The probability that an element survives to time $\mathrm{T}_{j}$ is

$$
1-F\left(T_{j}\right)=\prod_{i=1}^{j}\left(1-p_{i}\right)
$$

The cumulative failure rate at time $\mathrm{T}_{j}$ is then:

$$
F\left(T_{j}\right)=1-\prod_{i=1}^{j}\left(1-p_{i}\right)
$$

This report was prepared as an accoumt of Goverument aponsored work Neither the United This report was prepared as an accoumt of Goverument aponsored work Neither
States, nor the Commission, nor any person acting on behsif of the Commission

A Makes any warranty or representation, expressed or implied, with respect to the accuof any information, apparatus, method, or process disclosed in this report may not infringe privately owned rights, or
B Assumes any liablities with respect to the use of, or for damages resulting from the use of any informatton, apparatus, method, or process disclosed in this report

loyee or contractor of the Commission, or employee of such contractor, to the extent that such employee or contractor of the Commission, or employee of such contractor prepares, disaeminates, or provides acceas to, any information pursuant to his employment or contract 
For small $\mathrm{F}_{1}$, this may be written approximately as:

$$
F\left(T_{j}\right) \approx \sum_{i=1}^{j} p_{i}
$$

A plot of $F\left(T_{j}\right)$ versus $T_{j}$ is the failure rate curve for the non-parametric case in which no failure model is assumed. The problem is to use these basic data to estimate the shape and scale parameters of the two-parameter Weibull model assuming that this is the model.

(4) Estimation of Weibull Parameters

The Weibul1 cumulative distribution function may be written:

$$
\text { where } \begin{aligned}
F(t) & =1-\exp \left(-t^{a} / b\right) \\
a & =\text { chape parameter } \\
b & =\text { scale parameter }
\end{aligned}
$$

An obvious method of estimating the two parameters is to first linearize the model (4.1).

$$
\begin{aligned}
& \ln [1-F(t)]=-t^{a} / b \\
& \ln \{-\ln [1-F(t)]\}=a \ln t-\ln b
\end{aligned}
$$

This suggests that, with $F\left(T_{j}\right)$ defined as in (3.3), one could plot $\ln \left\{-\ln \left[1-F\left(t_{j}\right)\right]\right\}$ versus $\ln \mathrm{T}_{j}$. This plot should be a straight line with a slope equal to the shape parameter $a$, and an intercept of $1 \mathrm{n} b$. 
Point estimates of the two parameters can be found by a simple procedure such as "eye-balling" a straight line through the data, and reading off the slope and intercept. Normally, however, one would like more than just the point estimates, and would also prefer that the estimation procedure be more objective.

An improvement on this approach consists in using Aitken's generalized least squares method to estimate the slope and intercept [4]. This idea was used by Krane [5] when fitting a polynomial through failure data, which is a generalization of this problem. It has also been used by the author on fuel element failure data for a number of years with good success.

However, there is a feature of this approach that is disturbing. To avoid singularity in the matrix, it is necessary to have $r_{i}>0$ in each interval. This necessitates combining intervals. This practice is disturbing when the last couple intervals, for example, both have $r_{i}=0$. One worries about combining these intervals with the last one in which a failure occurrea. Perhaps the fact that $r_{i}=0$ in both intervals is evidence that the weibull failure model does not provide a satisfactory fit to the data, a situation that may be disguised if the intervals are combined to too large an extent. To circumvent this difficulty, an estimation procedure was devised which permits estimation even when $r_{i}=0$ in some of the intervals. Further, the procedure allows one to check on the adequacy of the assumption that the underlying model is the Weibull model. The procedure is described in the next section.

\section{(5) Proposed Estimation Procedure}

The estimation procedure consists in finding that combination of shape and scale parameters which gives the "best" overall agreement between the observed numbers of failures in each interval and those predicted by the 
model. For a given value of the shape parameter $a$, the maximum likelihood estimate of the scale parameter, b, is found by summing the element lifetimes raised to the $a^{\text {th }}$ power for both the failed and non-failed elements, and dividing by the number of fallures. For the grouped data as given in Table (2.1), the estimate of $b$ for given a may be written

$$
b_{a}=\frac{\sum_{i=1}^{k} L_{i}\left(\frac{x_{i-1}^{a}+x_{i}^{a}}{2}\right)}{\sum_{i=1}^{k} r_{i}}
$$

Values are assigned the shape parameter and $b_{a}$ is computed for each $a$. Each combination of the shape and scale parameters is then used to find the predicted number of failures which will occur in each time interval. For the grouped data, the number predicted in the $i^{\text {th }}$ interval is

$$
E_{i}=m_{i}\left\{\exp \left(-\frac{x_{i-1}^{a}}{b_{a}}\right)-\exp \left(-\frac{x_{i}^{a}}{b_{a}}\right)\right\}
$$

The $E_{i}$ are to be compared with the $r_{i}$. The value of the shape parameter, $a$, and the corresponding maximum likelihood estimate, $b_{a}$, which result in the "best" agreement between the $E_{i}$ and the $r_{i}$ will be the estimates derived from this procedure.

A problem is how to measure this agreement. The $E_{i}$ values will generally be too small to permit valid application of a Chi-square procedure, i.e., one which regards the statistic:

$$
\sum \frac{\left(E_{i}-r_{i}\right)^{2}}{E_{i}}
$$

A search was made for an alternate method of measuring the exient of this agreement. 
It was decided to associate a probability, $\mathrm{i}_{i}$, with each combination $\left(E_{i}, r_{i}\right)$. When $r_{i}$ is equal to $E_{i}$, one shouid like to have $P_{i}$ equal to one, while as $r_{i}$ deviates from $E_{i}$ either on the low side or on the high side, $P_{i}$ should become progressively smaller, approaching zero as the discrepancy becomes larger and larger. This criterion has intuitive appeal, but will be more firmly motivated later in the paper.

To define $P_{i}$ specifically, assume that the number of failures, $r_{i}$, observed in interval $i$ is a random variable following the Poisson distribution with parameter $E_{i}$. With the large sample sizes and small probabilities encountered in the application under discussion, this is a reasonable assumption. Then, one can define a probability $P_{i}^{\prime}$ as follows:

$$
\left.\begin{array}{l}
\text { If } r_{i} \leq E_{i}, \quad P_{i}^{\prime}=\sum_{j=0}^{r_{i}} e^{-E_{i}}\left(E_{i}\right)^{j} / j ! \\
\text { if } r_{i}>E_{i}, \quad P_{i}^{\prime}=\sum_{j=r_{i}}^{\infty} e^{-E_{i}\left(E_{i}\right)^{j} / j !}
\end{array}\right\}
$$

Equations (5.3) define $P_{i}$ ' such that if less failures $\left(r_{i}\right)$ are observed than expected $\left(E_{i}\right)$ from the model, then the probability of observing this number $\left(r_{i}\right)$ or less given a mean of $E_{i}$ is caiculated. If more failures are observed $\left(r_{i}\right)$ than expected $\left(E_{i}\right)$, then the probability of observing $r_{i}$ or more failures given $E_{i}$ is calculated.

With the definition given by (5.3), large discrepancies between $E_{i}$ and $r_{i}$ will result in small values of $\mathrm{P}_{i}$ ', while small discrepancies result in large values of $P_{i}^{\prime}$. However, $P_{i}^{\prime}$ will not range from 0 to 1 as desired; the maximum value will depend on $r_{i}$. One can normalize these probabilities by dividing each $P_{i}^{\prime}$ by some factor, $F_{i}$, which is a function of $r_{i}$. Specifically, 


$$
\begin{aligned}
F_{i} & =\sum_{j=0}^{r_{i}} e^{-r_{i}}\left(r_{i}\right)^{j} / j !, r_{i}>0 \\
& =1 \text { for } r_{i}=0
\end{aligned}
$$

Then, defining $P_{i}=P_{i}^{\prime} / F_{i}$ gives a random variable with range from zero to one.

Having defined $P_{i}$ for each $i$, the problem is then to define a statistic which will combine the $P_{i}$ 's in some fashion to give an overall measure of agreement between the $E_{i}$ and the $r_{i}$. A statistic that suggests itself is

$$
S=-2 \sum_{i=1}^{k} \ln P_{i}
$$

The motivation for choosing $S$ comes from the fact that if $q_{i}$ is a random variable uniformly distributed $c n(0,1)$, and if $q_{i}$ and $q_{j}$ are independent for all $(i, j)$, then $-2 \sum_{i=1}^{k} 1 n q_{i}$ follows the Chi-square distribution with $2 k$ degrees of freedom [6]. This fact is used, as one application, when combining independent tests of significance to obtain an overall significance level. This is the application that suggested the use of s in (5.5).

It is recognized that even though $P_{i}$ is distributed over the interval $(0,1)$, it is not uniformly distributed over this interval. Thus, the $S$ statistic will not be distributed as Chi-square except, perhaps, as the $E_{i}$ become larger. More is said on this point in the next section. From an estimation viewpoint, the only interest at this point is to define $S$, and establish some sort of motivation for the definition. 
Having defined $S$, the estimation proceciure suggested is summarized in the following steps.

(1) Choose a series of values for the shape parameter, a, defined over some selected interval $\left(a_{1}, a_{2}\right)$.

(2) For each value of $a$, calculate $b_{a}$ from (5.1)

(3) For each combination of values $\left(a, b_{a}\right)$, calculate the $E_{i}$ from $(5.2)$

(4) For each $i$, calculate $P_{i}$ from $(5.4),(5.3)$, and $P_{i}=P_{i}{ }^{\prime} / F_{i}$.

(5) Calculate $S$ from (5.5)

(6) The estimates of the shape and scale parameters are the combination which results in a minimum $S$ value.

\section{(6) Limits of Uncertainty}

Having estimated the two parameters, it is important to nbtain a cnnfilence region for them. Further, one would like to know the uncertainty Essociated with a predicted failure rate at some time $t$. This portion of tive paper addresses itself to this question.

The approach to finding a confidence region for the parameters will be to find those combinations of values which result in $S$ values equal to some cricical value $S_{c}$. This critical value will be one which would be exceeded by chance less than $\alpha \%$ of the time if, in fact, the Weibull model with given parameters vere the true model. The resulting combinations of parameters will bound the required confidencc region.

If $S$ were distributed approximately as the Chi-square distribution with $2 \mathrm{k}$ degrees of freedom, it would be a simple matter to determine $S_{c}$ from an appropriate table of this distribution. However, for small values of the $I_{i}$, the assumption that the $P_{i}$ are approximately uniformiy distributed over $(0, i)$ is not valid, and hence, this approact. cannot bc used. 
An investigation was made to find how $S_{c}$ may be determined, especially for small $E_{i}$. The approach was to calculate an average $S$ value and $i t s$ variance for each of a series of $E_{i}$ values by $l$ isting the possible values for $r_{i}$ and their associated probabilities of occurrence. An example will serve to sllustrate the approach. $E_{1}$ is set equal to 0.5 in this example

Table (6.1)

With

\begin{tabular}{|c|c|c|c|c|}
\hline$r_{i}$ equals & probability q & $\mathrm{P}_{i}^{\prime}[$ from $(5.3)]$ & $\mathrm{P}_{i}[$ from $(5.4)]$ & $S_{i}=-2 \ln P_{i}$ \\
\hline 0 & .6065 & .6065 & .6065 & 1.00 \\
\hline 1 & .3033 & .3935 & .5346 & 1.25 \\
\hline 2 & .0758 & .0902 & .1332 & 4.03 \\
\hline 3 & .0126 & .0144 & .0223 & 7.61 \\
\hline 4 & .00158 & .00175 & .00278 & 11.77 \\
\hline 5 & .000153 & .000172 & .000279 & 16.37 \\
\hline 6 & .000013 & .000014 & .000023 & 21.36 \\
\hline$\geq 7$ & 0 & & & \\
\hline
\end{tabular}

Columns 2 and 5 give the distribution of $s_{i}$ for one of the $k$ intervals. Operating on these two columns gives the first two monents for $s$ at $E_{i}=0.5$.

$$
\begin{aligned}
\text { Average } S & =1.408 \\
\text { Variance } S & =1.33
\end{aligned}
$$

Note that if $S$ were truly distributed as Chi-square with two degrees of freedom, its mean would be 2 and its variance 4. An inspection of colunns 2 and 4 in Table (6.1) illustrates how grossly the assumption that $P_{i}$ is uniformly distributed on $(0,1)$ is violated for $E_{i}=0.5$. It is not surprising in this case that the everage and variance of $S$ are quite different from what would be expected from the Chi-square distribution.

The two momith ware calculated for values of $E_{i}$ ranging from 0.1 to 10 . A plot of the nean and variance of $S$ vcrsus $E$ is given in figure ( $\epsilon .1)$. Note 
that the mean and variance gradually approach the limiting values of two and four respectively as $\mathrm{E}$ increases in value.

Having determined these moments as a function of $E$, one can obtain the moments for the sum of the $S_{i}$ over the $k$ intervals, recognizing that the $s_{1}$ are independently distributed. An appeal can then be made to the Central Limit Theorem to assert that the sum, $S$, is approximately normally distributed, and hence obtain a value for $S_{c}$ from a table of this distribution once $\alpha$ is specified.

This procedure was considered sufficiently precise for the application under discussion. If greater precision were required, one could find the higher moments of $S$ and appeal to some family of curves such as the Pearson or Johnson curves to determine $S_{c}$. This work is under consideration at present.

Cince the confirience region for the shape and scale parameters is found, the limits on a predicted failure rate at a given time $t$ are found by sweeping around the extremities of this region and locating the conbinations of the parameters which yield the minimum and maximum values for the iailure rate. This is done for a series of time values to give a confidence region for the failure rate curve.

It should be noted that the size of the confidence region depends not only on the number of failures observed, but it is also affected by the reasonableness of the underlying assumption that the Weibull function is applicable. If this assumption is not valid, it may well be that even the mininum value for $S$ is larger than the critical valus, suggesting that no combination of the Weibull parameters will provide an adequate fit. 


\section{(7) Numerical Example}

A numerical example of the estimation procedure is given to illustrate the method. The failure data are listed.

Table (7.1)

Failure data for example

\begin{tabular}{|c|c|c|c|}
\hline Time* & $\mathrm{N}_{i}$ & $\mathrm{~L}_{1}$ & $r_{i}$ \\
\hline $0-500$ & 6056 & 514 & 0 \\
\hline $500-600$ & 5542 & 968 & 2 \\
\hline $600-700$ & 4574 & 1310 & 2 \\
\hline $700-800$ & 3264 & 1154 & 2 \\
\hline $800-900$ & 2110 & 818 & 3 \\
\hline $500-1000$ & 1292 & 565 & 0 \\
\hline $1000-1100$ & 727 & 412 & 0 \\
\hline $1100-1200$ & 315 & 315 & 0 \\
\hline
\end{tabular}

The computer program varied the shape parameter a from 1.0 to 10.0 in increments of 0.2 . The minimum $S$ value (5.5) was found to occur at $a=4.0$. Values of $E_{i}$ and $P_{i}$ are given in Table (7.2) for this value of $\underline{a}$ and the associated maximum likelihood value of $b=2.906 \times 10^{14}$.

Table $(7.2)$

$\begin{array}{cccc}\text { Time } & \frac{E_{i}}{1.25} & \frac{I_{i}}{0} & \frac{P_{i}}{0-500} \\ 500-600 & 1.16 & 2 & 0.48 \\ 600-700 & 1.48 & 2 & 0.64 \\ 700-800 & 1.55 & 2 & 0.68 \\ 800-900 & 1.43 & 3 & 0.27 \\ 900-1000 & 1.18 & 0 & 0.31 \\ 1000-1100 & 0.82 & 0 & 0.44 \\ 1100-1200 & 0.33 & 0 & 0.72 \\ \mathrm{~S}=-2 \sum 1 \mathrm{n} \mathrm{P}_{i}=12.93 & \end{array}$


Reference to figure (6.1) gives the first two moments for $S$ for the $E_{1}$ values in Table $(7.2)$.

$$
\begin{aligned}
\text { Mean of } S & =13.05 \\
\text { Standard Deviation } & =4.02
\end{aligned}
$$

From a table of the normal distribution, $S$ exceeds $13.05+(1.282)(4.02)=$ 18.20 with a probability of 0.10 . Therefore, the $90 \%$ limits on the shape parameter are given by finding the smallest and largest values of the parameter which give values of $\mathrm{S}$ equal to 18.20. These are:

$$
\begin{array}{ll}
\text { lower limit: } & a=2.1 \\
\text { upper limit: } & a=6.5
\end{array}
$$

Finally, $90 \%$ limits of unccrtainty for the predicted failure rate are reproduced in Table ( 7.3 ).

\begin{tabular}{|c|c|c|c|}
\hline$t$ & $F(t) \times 10^{-4}$ & Lower Limit $\times 10^{-4}$ & Upper Limit $\dot{x} 10^{-4}$ \\
\hline 500 & 2.15 & 0.44 & 5.57 \\
\hline 600 & 4.46 & 1.41 & 10.94 \\
\hline 700 & 8.26 & 3.78 & 16.84 \\
\hline 800 & 14.09 & 8.89 & 25.63 \\
\hline 900 & 22.55 & 16.70 & 40.86 \\
\hline 1000 & 34.35 & 24.16 & 63.52 \\
\hline 1100 & 50.26 & 31.57 & 99.22 \\
\hline 1200 & 71.10 & 38.22 & 152.89 \\
\hline
\end{tabular}

Table (7.3)

Confidence iimits on predicted failure rates 
(8) Acknowledgement

The assistance of Mrs. Barbara B. Vinson who programmed this method for the computer is gratefully acknowledged.

\section{$\underline{\text { References }}$}

[1] Weibull, W., "A Statistical Distribution of Wide Applicability", Jour. of App1. Mech. 19, 109-13, 1951.

[2] Splitstone, Douglas, "Estimation of the Weibull Shape and Scale Parameters", Iowa State M.S. thesis, 1967.

[3] Kaplan, E. L., and Meier, Paul, "Nonparametric Estimation from Incomplete Observations", Jour. Amer. Stat. Assoc., 53, No. 282, 457-81, 1958.

(4] Aitlen, A. C., "On Least Squares and Linear Combinations of observaticns", Proc. Royal Snciety of Edinburgh, 55, 42-48, 1535.

[5] Krane, Scott A., "Analysis of Survival Data by Regression Techniques", Technometrics, 5, No. 2, 161-74, 1963.

[6] Anderson, R. L., and Bancroft, T. A., "Statistical Theory in Research", McGraw - Hill, p. ituo. 
